\section{P111 FLUTICASONE PROPIONATE/FORMOTEROL FUMARATE COMBINATION THERAPY IS SUPERIOR TO FLUTICASONE PROPIONATE ALONE IN IMPROVING ASTHMA CONTROL}

doi:10.1136/thoraxjnl-2011-201054c.111

${ }^{1} \mathrm{D}$ Price, ${ }^{2} \mathrm{~A}$ Papi, ${ }^{3} \mathrm{M}$ Tamm, ${ }^{4} \mathrm{~K}$ Kaiser, ${ }^{5} \mathrm{~B}$ Grothe, ${ }^{5} \mathrm{M}$ Lomax, ${ }^{5} \mathrm{~T}$ Mclver. ${ }^{1}$ Centre of Academic Primary Care, University of Aberdeen, Aberdeen, UK; ${ }^{2}$ Research Centre on Asthma and COPD, Department of Clinical and Experimental Medicine, University of Ferrara, Ferrara, Italy; ${ }^{3}$ Department of Pneumology, University Hospital, Basel, Switzerland; ${ }^{4}$ SkyePharma, Muttenz, Switzerland; ${ }^{5}$ Mundipharma Research Ltd., Cambridge, UK

Introduction and Objectives Poor asthma control impacts on patients' health and reduces their quality of life. Despite a rise in the proportion of patients receiving treatment, asthma remains uncontrolled in many patients. A new therapy option combining the inhaled corticosteroid fluticasone propionate (FLUT) with the long-acting $\beta_{2}$-agonist formoterol fumarate (FORM) in a single aerosol inhaler (FLUT/FORM; flutiform $^{\circledR}$ ) has been developed for the treatment of asthma. This integrated analysis of data from five randomised, double-blind, parallel-group phase 3 studies assessed the effects of FLUT/FORM compared with FLUT alone on the percentage of asthma control days.

Methods Symptomatic adult and adolescent patients with mild, moderate or severe asthma were randomly assigned to receive FLUT/FORM (100/10, $250 / 10$ or $500 / 20 \mu \mathrm{g}$ twice daily) or the equivalent nominal dose of FLUT alone $(100,250$ or $500 \mu \mathrm{g}$ twice daily) for 8 or 12 weeks. Most patients randomised to treatment had uncontrolled asthma. The percentage change in asthma control days (defined as those with no use of rescue medication, an asthma symptom score reporting no symptoms and a sleep disturbance score indicating that the patient slept through the night) was assessed from baseline to study end.

Results At baseline, the percentage of asthma control days was low in both groups (FLUT/FORM [ $n=623]$ : 12.8\%; FLUT [ $n=623]$ : $12.6 \%)$. FLUT/FORM combination therapy was superior to FLUT alone for improvement in percentage of asthma control days. At study end, patients in the FLUT/FORM group experienced a mean of $62.4 \%$ asthma control days, an improvement of $49.6 \%$ from baseline, whereas FLUT treatment was associated with $54.8 \%$ asthma control days (42.2\% change from baseline). FLUT/FORM provided a statistically significant improvement in asthma control days compared with FLUT alone (least-squares mean difference $[95 \%$ CI] : 7.5\% [3.21 to 11.84]; $\mathrm{p}<0.001)$.

Conclusions Fluticasone/formoterol combination therapy is superior to fluticasone monotherapy for improving the percentage of asthma control days over 8 or 12 weeks' treatment in adults and adolescents with mild, moderate or severe asthma. These data suggest the combination of fluticasone and formoterol in a single aerosol inhaler will provide an effective option for asthma maintenance therapy.

\section{P112 FLUTICASONE PROPIONATE/FORMOTEROL FUMARATE COMBINATION THERAPY REDUCES THE RISK OF EXACERBATIONS COMPARED WITH ITS INDIVIDUAL COMPONENTS IN PATIENTS WITH ASTHMA}

doi:10.1136/thoraxjnl-2011-201054c.112

${ }^{1} \mathrm{~S}$ Dissanayake, ${ }^{2} \mathrm{~J}$ Sastre, ${ }^{3} \mathrm{~A}$ Papi, ${ }^{4} \mathrm{~K}$ Kaiser, ${ }^{1} \mathrm{~B}$ Grothe, ${ }^{1} \mathrm{M}$ Lomax, ${ }^{1} \mathrm{~T}$ Mclver. ${ }^{1}$ Mundipharma Research Limited, Cambridge, UK; ${ }^{2}$ Fundacion Jimenez Diaz, Madrid, Spain; ${ }^{3}$ University of Ferrara, Ferrara, Italy; ${ }^{4}$ SkyePharma, Muttenz, Switzerland

Introduction and Objectives Asthma remains uncontrolled in many patients, as indicated by exacerbations, deteriorating symptoms and impaired quality of life. A new option has been developed for the maintenance treatment of asthma, combining the inhaled corticosteroid fluticasone propionate (FLUT) with the long-acting $ß_{2}$-agonist formoterol fumarate (FORM) in a single aerosol inhaler (FLUT/ FORM; flutiform $^{\circledR}$ ). A pooled analysis of data from up to five randomised, double-blind, parallel-group phase 3 studies assessed the effects of FLUT/FORM on asthma exacerbations.

Methods Adults and adolescents with mild, moderate or severe asthma were randomised to FLUT/FORM (100/10, 250/10 or 500/ $20 \mu \mathrm{g}$ twice daily), the equivalent nominal dose of FLUT monotherapy $(100,250$ or $500 \mu \mathrm{g}$ twice daily; five studies) or FORM monotherapy (10 $\mu \mathrm{g}$ twice daily; three studies) for 8 or 12 weeks. The endpoints assessed were time to first exacerbation and proportion of patients experiencing an exacerbation. Exacerbations were defined as peak expiratory flow rate $>30 \%$ below baseline, awakening at night due to asthma, use of rescue medication 3-4 times per day (each on $=2$ consecutive days; mild-to-moderate exacerbation), need for additional therapy, or emergency visit or hospitalisation due to asthma (severe exacerbation).

Results Time to first exacerbation (any severity) was significantly longer with FLUT/FORM ( $n=641)$ than with FLUT $(n=643$; $\mathrm{p}=0.01$ ). Similarly, time to first exacerbation was significantly longer with FLUT/FORM $(n=341)$ than FORM $(n=345 ; p<0.001)$. Overall, the proportion of patients with any exacerbation was significantly lower with FLUT/FORM (18.2\%) than with FORM $(31.3 \% ; \mathrm{p}<0.001)$. Fewer patients experienced severe exacerbations with FLUT/FORM than FORM alone (FLUT/FORM 2.4\%; FORM 9.6\%; $\mathrm{p}<0.001$ ). Similarly, fewer patients experienced any exacerbation with FLUT/FORM (26.8\%) than with FLUT (32.8\%; p=0.02) and fewer patients experienced severe exacerbations with FLUT/ FORM than FLUT alone (FLUT/FORM 1.9\%; FLUT 2.8\%; $p=0.36$ ). Conclusions Fluticasone/formoterol significantly reduces the risk of asthma exacerbations compared with its individual components. Combination therapy with fluticasone and formoterol in a single aerosol inhaler may help to improve asthma control and reduce the risk of asthma exacerbations that can impair patients' quality of life.

\section{P113 THE COST-EFFECTIVENESS OF STEP DOWN FROM HIGH DOSE FLUTICASONE/SALMETEROL DRY POWDER OR SUSPENSION FORMULATIONS IN ASTHMA APPLIED TO THE UK SETTING}

doi:10.1136/thoraxjnl-2011-201054c.113

${ }^{1} \mathrm{P}$ L Paggiaro, ${ }^{2} \mathrm{G}$ Buseghin, ${ }^{2} \mathrm{G}$ Nicolini, ${ }^{2} \mathrm{~S}$ Patel, ${ }^{3} \mathrm{~S}$ lannazzo, ${ }^{3} \mathrm{O}$ Zaniolo, ${ }^{4} \mathrm{~A}$ Papi. ${ }^{1}$ University of Pisa, Pisa, Italy; ${ }^{2}$ Chiesi Farmaceutici SpA, Parma, Italy; ${ }^{3}$ Adres Health Economics \& Outcomes Research, Turin, Italy; ${ }^{4}$ University of Ferrara, Ferrara, Italy

Objectives The stepwise management of asthma outlined in the BTS/SIGN guideline recommends stepping down asthma patients where control is achieved. The aim of this analysis was to estimate the costs and health outcomes associated with step down of controlled patients on high dose fluticasone/salmeterol (FP/S 1000/ $100 \mu \mathrm{g}$ daily) to either extrafine beclometasone/formoterol (BDP/F $400 / 24 \mu \mathrm{g})$ pMDI or medium dose $\mathrm{FP} / \mathrm{S}(500 / 100 \mu \mathrm{g})$ dry powder in the UK setting.

Methods A patient-level simulation Markov model was constructed to enable the simulation of a cohort of patients through three comparative arms (FP/S 1000/100, FP/S 500/100, BDP/F 400/24). Transition probabilities and healthcare resources costs were derived from a recent multinational clinical trial comparing BDP/F 400/ $24 \mu \mathrm{g}$ vs FP/S 500/100 $\mu \mathrm{g}$ as step down therapy in asthma. Direct costs and health state utilities were sourced from UK costs and published literature. The cost of FP/S 1000/100 was calculated considering the dry powder formulation. An additional analysis was conducted considering the suspension formulation. The analysis was conducted from a UK health system perspective, based on 6 months horizon. Probabilistic sensitivity analyses were conducted. 
Results The analysis showed that there was an ICER (Incremental Cost-Effectiveness Ratio) of 32000 GBP/OALY (Quality Adjusted Life Years) associated with high dose dry powder FP/S 1000/100 $\mu \mathrm{g}$ vs extrafine BDP/F 400/24 $\mu \mathrm{g}$ and an ICER of approximately 36800 GBP/OALY associated with medium dose dry powder FP/S 500/ $100 \mu \mathrm{g} v \mathrm{~s}$ BDP/F 400/24 $\mu \mathrm{g}$. Additional analysis showed that there was an ICER of 85200 GBP/OALY associated with high dose suspension formulation $\mathrm{FP} / \mathrm{S}$ 1000/100 $\mu \mathrm{g}$ vs extrafine BDP/F 400/ $24 \mu \mathrm{g}$.

Conclusions BTS/SIGN guideline recommend that when asthma control is achieved, treatment can be stepped down to the lowest dose that maintains control. It was found that maintaining controlled patients on high dose FP/S is not cost-effective. Extrafine $\mathrm{BDP} / \mathrm{F} 400 / 24 \mu \mathrm{g}$ daily can be considered to be a cost-effective option in the UK to maintain control of asthmatic patients stepped down from high dose $\mathrm{FP} / \mathrm{S} 1000 / 100 \mu \mathrm{g}$ daily dry powder or suspension formulations and the magnitude of cost effectiveness is estimated to be highest when stepping down from the suspension formulation.

\section{P114 DIFFICULT ASTHMA: THE PLYMOUTH EXPERIENCE}

doi:10.1136/thoraxjnl-2011-201054c.114

B Castell, D Pike, M Masoli. Plymouth Hospitals NHS Trust, Plymouth, Devon

Introduction Difficult asthma represents a significant unmet clinical need and burden on healthcare resources. We recently set up a difficult asthma clinic (DAC) in Plymouth and conducted an observational study of our experience to date.

Methods The DAC started in April 2010 evaluating patients using a systematic multidisciplinary approach. Patients were symptomatic at $=$ Step 4 BTS guidelines and arose from a combination of cross referrals, inherited patients and asthmatics under regular chest clinic follow-up.

Results 113 patients were evaluated within the DAC. 74 women. Mean age 48 yrs. 15 patients were felt either not to have asthma or asthma was not the primary diagnosis. Diagnoses included bronchiectasis (3), Goitre (1), obliterative bronchiolitis (1), tracheal involvement from ulcerative colitis (1), chronic pulmonary emboli (1), Churg-Strauss syndrome (1). 98 patients had difficult asthma. 14 patients had an occupational element, three with reactive airways dysfunction syndrome. 72 had comorbidities which included: vocal cord dysfunction/dysfunctional breathing (19), bronchiectasis (20), Class II Obesity (19), COPD/emphysema (9), GORD (31), immune deficiency (5), OSA (5), psychological (11), allergic bronchopulmonary aspergillosis (2). Medication at baseline: 46 patients were on long term oral corticosteroids (OCS) (mean $22 \mathrm{mg} / \mathrm{d}$ ). Most patients were able to significantly reduce their OCS dose, mean reduction $53 \%$. 12 were able to discontinue OCS entirely. Mean inhaled corticosteroid dose $2287 \mathrm{mcg} / \mathrm{d}$ (BDP equivalent). Subcutaneous terbutaline (3), cyclosporin (2), Anti-IgE therapy (1). Currently seven on anti-IgE therapy. 58 had severe refractory asthma by American Thoracic Society criteria. Mean IgE $531 \mathrm{kU} / \mathrm{l}$, mean FeNO $40.5 \mathrm{ppb} .15 / 58$ had fungal sensitivity. Adherence: 1 of 12 patients tested was identified as non-adherent with undetectable prednisolone level and normal cortisol. Healthcare utilisation: 68 patients with 12 months follow-up data demonstrated a significant reduction in hospitalisations compared to the previous 12 months, 1.00 vs 0.53 .

Conclusion This study highlights the importance of alternative diagnoses and comorbidities in the work up of difficult asthma. IgE and FeNO were higher than expected as was adherence to OCS compared to published studies. The implementation of a DAC has reduced hospital admissions, reduced OCS requirement and enhanced access to treatments such as Anti-IgE therapy.

\section{Cellular responses in the aetiology of COPD P115 CHRONIC DIESEL EXHAUST PARTICLE (DEP) EXPOSURE DIFFERENTIALLY ALTERS MONOCYTE DIFFERENTIATION AND FUNCTION IN HEALTHY CONTROLS COMPARED TO COPD}

doi:10.1136/thoraxjnl-2011-201054c.115

${ }^{1} \mathrm{~N}$ Chaudhuri, ${ }^{2} \mathrm{H}$ Jary, ${ }^{3} \mathrm{~S}$ Lea, ${ }^{3} \mathrm{~N}$ Khan, ${ }^{1} \mathrm{~L} \mathrm{C}$ Parker, ${ }^{3} \mathrm{D}$ Singh, ${ }^{1} \mathrm{I}$ Sabroe. ${ }^{1}$ Academic Unit of Respiratory Medicine, University of Sheffield, Sheffield, UK; ${ }^{2}$ Academic Unit of Respiratory Medicine, The University of Newcastle, Newcastle, UK; ${ }^{3}$ Respiratory Medicine Research Group, University of Manchester, Manchester, UK

Introduction and Objectives Alveolar macrophages are heavily implicated in the pathogenesis COPD. During chronic inflammation, macrophages mature continuously from infiltrating monocytes that are continually recruited to the airways. We have previously found DEP modulate life span and function of monocytes from healthy donors, but their effects on monocytes of people with COPD are unknown, and were therefore the subject of this study.

Methods Monocytes were purified from the blood of patients with GOLD II/III COPD and healthy age matched controls Monocytederived macrophages (MDMs) were generated in the presence or absence of DEP and their lifespan studied. Cytokine generation in response to TLR agonists and heat killed bacteria was assessed by ELISA and expression of CD14 was measured by FACS.

Results Chronic exposure of monocytes from patients with COPD to DEP concentrations above $10 \mu \mathrm{g} / \mathrm{ml}$ caused a significant reduction in cell survival. Lower concentration of chronic DEP exposure, as low as $1 \mu \mathrm{g} / \mathrm{ml}$, caused impairment of cytokine responses to LPS and heat killed Escherichia Coli, and this phenotype was associated with a reduction in CD14 surface marker expression. However, COPD monocytes were generally more resistant to the effects of DEP compared to healthy control cells.

Conclusions In this study monocytes from healthy volunteers appeared to be more susceptible to the harmful effects of chronic DEP exposure compared to those from individuals with COPD. These findings reinforce the evidence that circulating leukocytes in COPD patients have altered phenotypes.

\section{P116 TESTING ANTIOXIDANT AND ANTI-INFLAMMATORY THERAPIES IN A COMPLEX LUNG TISSUE MODEL}

doi:10.1136/thoraxjnl-2011-201054c.116

T S Singh, F R Razali, M N North, T W Wilkinson, B L N Nicholas. University of Southampton, School of medicine, Southampton, UK

COPD is a disease of global importance and its primary cause airway inflammation as a consequence of cigarette smoking is well described. However, there remains a lack ofeffective therapies for this important condition. Animal models of disease are limited in their predictive utility and therefore creation of a complex, human disease modelis an important step for testing new therapeutic interventions. We therefore established a tissue model of oxidative and inflammatory responses to relevant triggers-cigarette smoke and LPS and determined the impact of interventions in the optimised system.

Methods Human lung tissueexplants from the resected lobes of six consented patients undergoing lobectomy were used. Uniform tissue explants were established on a novel culture system and then treated with CSE and LPS before the supernatants were collected optimal dosing was determined. Treatments and control experiments were performed with the anti-oxidant Vitamin $\mathrm{C}$ and fluticasone. Inflammatory readouts were measured by ELISA; TNFa, IL-8 and MMP-9. 